\title{
Reliability Assessment for Distribution Optimization Models: A Non-Simulation-Based Linear Programming Approach
}

\author{
Gregorio Muñoz-Delgado, Student Member, IEEE, Javier Contreras, Fellow, IEEE, and \\ José M. Arroyo, Senior Member, IEEE
}

\begin{abstract}
The advent of smart grids and active distribution networks has boosted the relevance of reliability in the operation and planning of distribution systems. As is customary, reliability is assessed analytically through several standard indices. Unfortunately, analytical reliability assessment relies on simulation, thereby requiring the use of inexact heuristic- or metaheuristicbased solution methods to operate and plan distribution systems when economic and reliability criteria are jointly considered. In order to overcome this shortcoming, this paper presents a new optimization-based approach to compute the standard network-dependent reliability indices that are widely used in reliability-constrained distribution optimization models. As a major salient feature over the conventional simulation-based method, reliability indices are equivalently determined by an efficient approach based on linear programming where the network topology is explicitly represented by decision variables of the optimization process. The proposed approach has been tested on several benchmarks including a 1080-node system. Numerical simulations show that the proposed approach yields the same results as the conventional algorithm. Moreover, the moderate computational effort is suitable for the subsequent integration of the proposed equivalent formulation in reliability-constrained optimization models for distribution operation and planning. Such successful numerical experience backs the potential of the proposed formulation to enable the use of sound techniques different from the available heuristics and metaheuristics to solve reliability-constrained operational and planning optimization models for distribution systems.
\end{abstract}

Index Terms-Analytical reliability assessment, distribution optimization models, linear programming, non-simulation-based approach.

\section{NOMENCLATURE}

A. Indices

$b$

Index for the load levels used to represent the loading condition.

$i, j, r, s, s^{\prime} \quad$ Indices for nodes.

B. Sets

$B$

Index set of the load levels used to represent the loading condition.

$\Upsilon$

Set of branches.

$\Psi_{i} \quad$ Index set of nodes connected to node $i$.

This work was supported in part by the Ministry of Economy and Competitiveness of Spain, under Project ENE2015-63879-R (MINECO/FEDER, UE), and the Junta de Comunidades de Castilla-La Mancha, under Project POII-2014-012-P and Grant PRE2014/8064.

The authors are with the Escuela Técnica Superior de Ingenieros Industriales, Universidad de Castilla-La Mancha, 13071 Ciudad Real, Spain (e-mail: gregorio.munoz.delgado@gmail.com; Javier.Contreras@uclm.es; JoseManuel.Arroyo@uclm.es).

$$
\begin{aligned}
& \Psi^{L N} \\
& \Psi^{N} \\
& \Psi_{i j}^{R P} \\
& \\
& \Psi^{S S} \\
& \Psi_{i j}^{S W}
\end{aligned}
$$
$L N$$$
\text { ij }
$$

C. Parameters

$A S A I$

$C I D_{s}$

$C I F_{s}$

$D_{i j}^{C B, S W}$

$D_{s}^{R P}$

$D_{s}^{S W}$

$D_{s}^{T, S W}$

$\bar{D}_{s}^{S W}$

EENS

$\ell_{i j}$

$L_{s}$

$\tilde{L}_{i s}$

$N_{i j}^{C B}$

$N_{s}^{R P}$

$N_{s}^{S W}$

$N_{s}^{T}$

$N C_{s}$

$S A I D I$

$S A I F I$
Index set of load nodes.

Index set of system nodes.

Index set of nodes affected by a repair-andswitching interruption due to a fault in the branch connecting nodes $i$ and $j$.

Index set of substation nodes.

Index set of nodes affected by a switchingonly interruption due to a fault in the branch connecting nodes $i$ and $j$.

Average system availability index.

Customer interruption duration at node $s$.

Customer interruption frequency at node $s$.

Value of $D_{s}^{T, S W}$ for all the nodes protected by the circuit breaker located at the branch connecting nodes $i$ and $j$.

Expected duration of repair-and-switching interruptions affecting node $s$.

Expected duration of switching-only interruptions affecting node $s$.

Parameter used to compute $D_{s}^{S W}$.

Parameter used to compute $D_{s}^{S W}$.

Expected energy not supplied.

Length of the branch connecting nodes $i$ and $j$.

Peak demand at node $s$.

Demand at node $i$ of the fictitious system under the operating condition corresponding to node $s$.

Total expected rate of interruptions affecting the circuit breaker located at the branch connecting nodes $i$ and $j$.

Expected rate of repair-and-switching interruptions affecting node $s$.

Expected rate of switching-only interruptions affecting node $s$.

Total expected rate of interruptions affecting node $s$.

Number of customers at node $s$.

System average interruption duration index.

System average interruption frequency index. 


$\begin{array}{ll}\Delta_{b} & \begin{array}{l}\text { Duration of load level } b . \\ \lambda_{i j}\end{array} \\ & \text { Failure rate of the branch connecting nodes } i \\ & \text { and } j . \\ \mu_{b} & \text { Loading factor of load level } b . \\ \tau_{i j}^{R P} & \text { Duration of the repair-and-switching interrup- } \\ & \text { tions associated with the failure of the branch } \\ & \text { connecting nodes } i \text { and } j . \\ \tau_{i j}^{S W} & \begin{array}{l}\text { Duration of the switching-only interruptions } \\ \text { associated with the failure of the branch con- } \\ \end{array} \\ & \text { necting nodes } i \text { and } j .\end{array}$

D. Variables

$\tilde{f}_{i j s} \quad$ Variable used to represent the power flow through the branch connecting nodes $i$ and $j$ of the fictitious system under the operating condition corresponding to node $s$.

$\tilde{g}_{i s}^{S S} \quad$ Power injection at substation node $i$ of the fictitious system under the operating condition corresponding to node $s$.

\section{INTRODUCTION}

$\mathbf{R}$ ELIABILITY is defined as the ability of a power system to continuously meet the electricity needs of end users with the required quantity and quality [1]. Reliability in distribution systems is fundamental since, as reported in the literature [2], more than $80 \%$ of all customer interruptions are due to failures at this level. Moreover, the new context where smart grids come into play has drastically changed the traditional passive role of distribution networks, leading to the paradigm of active distribution networks. Therefore, an adequate consideration of reliability in distribution system operation and planning is essential.

According to [2]-[8], several indices are available to quantitatively measure system reliability such as customer interruption frequency (CIF), customer interruption duration (CID), system average interruption frequency index (SAIFI), system average interruption duration index (SAIDI), average system availability index (ASAI), and expected energy not supplied (EENS). A relevant and practical technique to calculate such indices is the analytical predictive method [1], [2], [7], [9]. For a given network topology, the analytical method quantifies the impact of a pre-specified set of events on service continuity through the simulation of component outages, one at a time. To that end, two pieces of information are used to compute standard reliability indices, namely failure rates and interruption durations.

The simulation-based nature and the topology dependence of the analytical reliability assessment have motivated the use of approximate methods to explicitly incorporate the computation of reliability indices in operational and planning models where topology decisions are outcomes of the optimization process. A widely-used method relies on metaheuristics to iteratively handle a population of candidate topologies [10], [11]. For each candidate topology, the conventional simulationbased algorithm is run. Hence, the impact of reliability on topology decisions drives the exploration of the search space. Unfortunately, metaheuristics are unable to identify whether global optimality has been attained. An alternative method was recently presented in [8], wherein a pool of candidate topologies was generated through an iterative algorithm based on standard mathematical programming with no regard to reliability. Subsequently, reliability was calculated ex post in order to make informed decisions. This heuristic decouples topology decisions from reliability, thereby giving rise to an inexact approach. Therefore, new non-heuristic approaches for the incorporation of reliability in distribution operation and planning are yet to be explored.

Motivated by the lack of exact methods for reliabilityconstrained distribution optimization problems and the appealing features of linear programming, the main contribution of this paper is the formulation of a novel approach based on linear programming for the analytical reliability assessment of a distribution system. Note that linear programming provides a sound mathematical programming framework with wellknown properties in terms of convergence and solution quality. Moreover, off-the-shelf software is readily available, which is beneficial for practical implementation purposes. To the best of the authors' knowledge, this is the first attempt in the literature to use an optimization process to equivalently implement the analytical simulation-based reliability assessment. The proposed approach relies on the solution of a set of optimal power flows, one per load node, for a topologically identical fictitious system. Each optimal power flow is formulated as a linear program associated with a pre-specified operating condition. As a result, the network topology is explicitly characterized through the outcomes of the optimization. Hence, two relevant advantages are featured over the traditional algorithmic calculation of reliability: 1) the proposed model paves the way for addressing reliability-constrained operational and planning models without requiring heuristics or metaheuristics, and 2) the use of linear programming provides an effective framework for such incorporation of reliability.

The remainder of this paper is organized as follows. Section II presents the main aspects of the analytical reliability evaluation. Section III describes the proposed approach and its potencial applicability. In Section IV, numerical results from several case studies are reported and analyzed. Relevant conclusions are drawn in Section V. Finally, the Appendix provides a general formulation for the reliability-constrained distribution optimization models resulting from the application of the proposed approach for reliability assessment.

\section{Analytical Reliability Assessment}

Distribution system reliability has been widely assessed on a yearly basis through well-known metrics such as CIF, CID, SAIFI, SAIDI, ASAI, and EENS [2]-[8]. CIF and CID account for the frequency and the duration of interruptions at each load node, respectively. SAIFI is a measure of how many sustained interruptions an average customer will experience. SAIDI is a measure of how many interruption hours an average customer will experience. ASAI is defined as the proportion of hours that the service will be available for an average customer. Finally, EENS represents the total energy that is expected to be curtailed. Next, the analytical reliability assessment typically implemented in distribution optimization models is described. 


\section{A. Reliability Indices in Distribution Optimization Models}

In reliability-constrained optimization models for distribution systems [8], [12]-[21], the calculation of standard reliability indices relies on the knowledge of the network topology and the loading condition. Within this optimizationbased framework, the following practical assumptions are customarily adopted for the sake of tractability [8], [12]-[21]:

1) Only sustained interruptions due to single branch outages are considered. Branch outages are characterized by failure rates and interruption durations.

2) A radially-operated distribution system is considered wherein each branch connected to a substation is equipped with a circuit breaker without a recloser at the output of the substation. Moreover, all branches are equipped with a switch that enables the isolation of the part of the system downstream of the fault in order to meet the demand of the healthy portion of the system. Thus, once a sustained fault has occurred, the first circuit breaker upstream of the fault trips, thereby curtailing all downstream load demands. Subsequently, the system topology is reconfigured by operating switches and circuit breakers to reduce the non-supplied energy. To that end, the first switch upstream of the fault is opened in order to isolate the fault. Then the circuit breaker is closed so that the supply to all load demands between the circuit breaker and the switch is restored. Finally, once the isolated fault is cleared, the corresponding switch is closed and complete service is reestablished.

Load nodes are thus affected by two types of interruption: 1) repair-and-switching interruptions, for which the supply is not restored until the damage is repaired, and 2) switching-only interruptions, which are associated with the network reconfiguration implemented to clear a faulty component.

Admittedly, a complete assessment of reliability should consider temporary faults, protection failures, transient disturbances, line overloading, and additional post-fault network reconfiguration to restore the service for load nodes downstream of the fault. This generalization would, however, render the problem essentially intractable through optimization. These modeling limitations notwithstanding, addressing reliabilityconstrained operational and planning models, albeit ignoring those practical aspects, is acceptable [8], [12]-[21] and provides the decision maker with a first estimate of a costeffective and reliable solution.

Under both assumptions, the impact of outages on load nodes can be quantified in a compact way in terms of the expected rate of nodal repair-and-switching interruptions, $N_{s}^{R P}$, the expected rate of nodal switching-only interruptions, $N_{S}^{S W}$, the expected duration of nodal repair-and-switching interruptions, $D_{s}^{R P}$, and the expected duration of nodal switching-only interruptions, $D_{s}^{S W}$, which are computed as:

$N_{s}^{R P}=\sum_{(i, j) \in \Upsilon \mid s \in \Psi_{i j}^{R P}} \lambda_{i j} ; \forall s \in \Psi^{L N}$

$$
\begin{aligned}
N_{s}^{S W} & =\sum_{(i, j) \in \Upsilon \mid s \in \Psi_{i j}^{S W}} \lambda_{i j} ; \forall s \in \Psi^{L N} \\
D_{s}^{R P} & =\sum_{(i, j) \in \Upsilon \mid s \in \Psi_{i j}^{R P}} \lambda_{i j} \tau_{i j}^{R P} ; \forall s \in \Psi^{L N} \\
D_{s}^{S W} & =\sum_{(i, j) \in \Upsilon \mid s \in \Psi_{i j}^{S W}} \lambda_{i j} \tau_{i j}^{S W} ; \forall s \in \Psi^{L N} .
\end{aligned}
$$

Moreover, reliability indices can be mathematically expressed as follows:

$$
\begin{aligned}
& C I F_{s}=N_{s}^{R P}+N_{s}^{S W} ; \forall s \in \Psi^{L N} \\
& C I D_{s}=D_{s}^{R P}+D_{s}^{S W} ; \forall s \in \Psi^{L N} \\
& S A I F I=\frac{\sum_{s \in \Psi^{L N}} N C_{s}\left(N_{s}^{R P}+N_{s}^{S W}\right)}{\sum_{s \in \Psi^{L N}} N C_{s}} \\
& S A I D I=\frac{\sum_{s \in \Psi^{L N}} N C_{s}\left(D_{s}^{R P}+D_{s}^{S W}\right)}{\sum_{s \in \Psi^{L N}} N C_{s}} \\
& A S A I=1-\frac{S A I D I}{8760} \sum_{b} \sum_{b \in B} C I D_{s} \mu_{b} L_{s} . \\
& E E N S=\sum_{s \in \Psi^{L N}} \frac{\Delta_{b}}{8760}
\end{aligned}
$$

\section{B. Simulation-Based Algorithm}

For a given network topology, the expected rates of nodal interruptions, $N_{s}^{R P}$ and $N_{s}^{S W}$, and the expected durations of nodal interruptions, $D_{s}^{R P}$ and $D_{s}^{S W}$, are conventionally calculated using a simulation-based algorithm [7] where outages of system branches are analyzed one at a time. For each branch, the expected rates of repair-and-switching and switching-only interruptions of those nodes affected by a fault in such a branch are increased by the corresponding failure rate. Similarly, the expected durations of repair-and-switching and switching-only interruptions of those nodes affected by a fault in such a branch are increased by the product of the corresponding failure rate and duration. This algorithm is outlined as follows:

Set $N_{s}^{R P}, N_{s}^{S W}, D_{s}^{R P}$, and $D_{s}^{S W}$ equal to 0 for all load nodes Loop over all branches $i-j$

Loop over all load nodes $s$

If node $s$ experiences a repair-and-switching interruption due to a fault in branch $i-j$

$$
\begin{aligned}
& N_{s}^{R P} \leftarrow N_{s}^{R P}+\lambda_{i j} \\
& D_{s}^{R P} \leftarrow D_{s}^{R P}+\lambda_{i j} \tau_{i j}^{R P}
\end{aligned}
$$

Elseif node s experiences a switching-only interruption due to a fault in branch $i-j$

\section{End if}

$$
\begin{aligned}
& N_{s}^{S W} \leftarrow N_{s}^{S W}+\lambda_{i j} \\
& D_{s}^{S W} \leftarrow D_{s}^{S W}+\lambda_{i j} \tau_{i j}^{S W}
\end{aligned}
$$

End loop

End loop

Once $N_{s}^{R P}, N_{s}^{S W}, D_{s}^{R P}$, and $D_{s}^{S W}$ are determined, reliability indices can be readily computed using (5)-(10). 


\section{Useful Remarks}

The exhaustive simulation of all possible branch outages for a given radially-operated topology yields a set of relevant remarks that form the basis of the novel optimization-based approach presented in Section III.

First, the following two remarks relating nodal interruption rates and branch failure rates can be made:

Remark 1): The expected rate of repair-and-switching interruptions affecting node $s, N_{s}^{R P}$, is equal to the sum of the failure rates of the branches used to supply the demand at this node from the substation node.

Remark 2): The expected rate of switching-only interruptions affecting node $s, N_{s}^{S W}$, is equal to the sum of the failure rates of those branches in the feeder including this node that are not used to supply the load at this node from the substation node. Hence, the expected rate of switching-only interruptions affecting a node can be calculated as the difference between the total expected rate of interruptions, $N_{s}^{T}$, and the expected rate of repair-and-switching interruptions, $N_{s}^{R P}$, affecting this node. The total expected rate of interruptions affecting node $s, N_{s}^{T}$, is equal to the sum of the failure rates of all branches belonging to the feeder where node $s$ is located, which is also the total expected rate of interruptions affecting the circuit breaker protecting that feeder, denoted by $N_{i j}^{C B}$. Note that, for the breaker located at the branch connecting nodes $i$ and $j, N_{i j}^{C B}$ is also equal to the sum over all nodes protected by this breaker of the failure rates of the branches injecting power at each node.

Additionally, two remarks associated with the expected durations of interruptions are described below:

Remark 3): The expected duration of repair-and-switching interruptions affecting node $s, D_{s}^{R P}$, is equal to the sum over the branches used to supply the demand at this node from the substation node of the products of the corresponding failure rate and repair-and-switching interruption duration.

Remark 4): The expected duration of switching-only interruptions affecting node $s, D_{s}^{S W}$, is equal to the sum over those branches in the feeder including this node that are not used to supply the load at this node from the substation node of the products of the corresponding failure rate and switching-only interruption duration. Similar to $N_{s}^{S W}, D_{s}^{S W}$ can be calculated as the difference between two parameters, namely $D_{s}^{T, S W}$ and $\bar{D}_{s}^{S W} . D_{s}^{T, S W}$ is equal to the sum over all branches belonging to the feeder where node $s$ is located of the products of the corresponding failure rate and switching-only interruption duration. Thus, for the breaker located at the branch connecting nodes $i$ and $j$, all $D_{s}^{T, S W}$ for the nodes in the feeder protected by that breaker are identical. Such a breaker-related expected duration is denoted by $D_{i j}^{C B, S W}$. Note that $D_{i j}^{C B, S W}$ is also equal to the sum over all nodes protected by this breaker of the products of the failure rates of the branches injecting power at each node and their corresponding switching-only interruption durations. In addition, $\bar{D}_{s}^{S W}$ is equal to the sum of the products of the failure rates of the branches used to supply the demand at node $s$ from the substation node and their corresponding switching-only interruption durations.

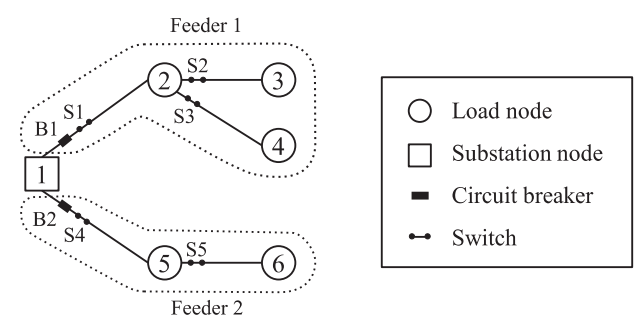

Fig. 1. Illustrative example.

\section{Illustrative Example}

The above-described analytical reliability assessment essentially consists in the calculation of the expected nodal interruption rates and durations, $N_{s}^{R P}, N_{s}^{S W}, D_{s}^{R P}$, and $D_{s}^{S W}$. Such calculation, which is typically implemented through the simulation-based algorithm described in Section II-B, is numerically illustrated with the radial system depicted in Fig. 1. This example comprises one substation node, node 1 , and five load nodes, nodes 2-6. The substation supplies two feeders with three and two branches, respectively. Branch failure rates, $\lambda_{12}, \lambda_{15}, \lambda_{23}, \lambda_{24}$, and $\lambda_{56}$, are equal to $0.5,0.3,0.2,0.1$, and 0.4 failures per year, respectively. Repair-and-switching interruption durations for each branch, $\tau_{12}^{R P}, \tau_{15}^{R P}, \tau_{23}^{R P}, \tau_{24}^{R P}$, and $\tau_{56}^{R P}$, are equal to $1.0,1.5,2.0,4.0$, and 3.0 hours per failure, respectively. Finally, switching-only interruption durations for each branch, $\tau_{12}^{S W}, \tau_{15}^{S W}, \tau_{23}^{S W}, \tau_{24}^{S W}$, and $\tau_{56}^{S W}$, are equal to $0.15,0.20,0.50,0.25$, and 0.60 hours per failure, respectively.

The impact of all single branch outages in terms of types of nodal interruptions is shown in Table I (columns 3-7). For expository purposes, two branch failures are analyzed in detail. Let us first consider a single fault at the branch connecting nodes 2 and 4 . This fault yields the automatic tripping of circuit breaker B1 and, hence, the loads connected at nodes 2-4 are curtailed. Next, switch S3 is opened to repair the fault and circuit breaker B1 is manually closed. Therefore, during the repair time, all loads are supplied except the load at node 4. When the fault is cleared, switch S3 is closed and normal operation is reestablished. As a result, the loads at nodes 2 and 3 are affected during the switching process, the load at node 4 is affected during the repair and switching periods, and the loads at nodes 5 and 6 , i.e., outside the feeder where the outage occurs, are not affected by this fault. This impact is reflected in the sixth column of Table I.

Similarly, as shown in the third column of Table I, if a fault occurs at the branch connecting nodes 1 and 2, loads at nodes 2-4 are affected during the repair and switching periods since such loads are downstream of the fault. In contrast, loads at nodes 5 and 6 are unaffected by this fault.

According to the simulation-based algorithm presented in Section II-B, the expected rates of repair-and-switching interruptions and switching-only interruptions affecting each node, which are listed in columns 8 and 9 of Table I, respectively, can be calculated adding the corresponding branch failure rates. Analogously, the expected durations of repair-and-switching interruptions and switching-only interruptions affecting each node, which are listed in columns 10 and 11 of Table I, respectively, can be calculated through the summation over 
TABLE I

ILLUSTRATIVE EXAMPLE - RESULTS

\begin{tabular}{|c|c|c|c|c|c|c|c|c|c|c|}
\hline \multirow{3}{*}{ Node } & \multicolumn{6}{|c|}{ Impact of failures } & \multirow{2}{*}{\multicolumn{2}{|c|}{$\begin{array}{l}\text { Expected rates of interruptions } \\
\text { (interruptions/year) }\end{array}$}} & \multirow{2}{*}{\multicolumn{2}{|c|}{$\begin{array}{l}\text { Expected durations of interruptions } \\
\text { (h/year) }\end{array}$}} \\
\hline & \multirow{2}{*}{$\begin{array}{c}\text { Type of } \\
\text { interruption }\end{array}$} & \multicolumn{5}{|c|}{ Out-of-service branch } & & & & \\
\hline & & $1-2$ & $1-5$ & $2-3$ & $2-4$ & $5-6$ & $N_{s}^{R P}$ & $N_{s}^{S W}$ & $D_{s}^{R P}$ & $D_{s}^{S W}$ \\
\hline \multirow{2}{*}{2} & Repair \& switching & $\checkmark$ & - & - & - & - & \multirow{2}{*}{0.5} & \multirow{2}{*}{0.3} & \multirow{2}{*}{0.500} & \multirow{2}{*}{0.125} \\
\hline & Switching only & - & - & $\checkmark$ & $\checkmark$ & - & & & & \\
\hline \multirow{2}{*}{3} & Repair \& switching & $\checkmark$ & - & $\checkmark$ & - & - & \multirow{2}{*}{0.7} & \multirow{2}{*}{0.1} & \multirow{2}{*}{0.900} & \multirow{2}{*}{0.025} \\
\hline & Switching only & - & - & - & $\checkmark$ & - & & & & \\
\hline \multirow{2}{*}{4} & Repair \& switching & $\bar{\checkmark}$ & - & - & $\bar{\checkmark}$ & - & \multirow{2}{*}{0.6} & \multirow{2}{*}{0.2} & \multirow{2}{*}{0.900} & \multirow{2}{*}{0.100} \\
\hline & Switching only & - & - & $\checkmark$ & - & - & & & & \\
\hline \multirow{2}{*}{5} & Repair \& switching & - & $\checkmark$ & - & - & - & \multirow{2}{*}{0.3} & \multirow{2}{*}{0.4} & \multirow{2}{*}{0.450} & \multirow{2}{*}{0.240} \\
\hline & Switching only & - & - & - & - & $\checkmark$ & & & & \\
\hline \multirow{2}{*}{6} & Repair \& switching & - & $\checkmark$ & - & - & $\checkmark$ & \multirow{2}{*}{0.7} & \multirow{2}{*}{0.0} & \multirow{2}{*}{1.650} & \multirow{2}{*}{0.000} \\
\hline & Switching only & - & - & - & - & - & & & & \\
\hline
\end{tabular}

the corresponding out-of-service branches of the products of the associated branch failure rate and outage duration.

This example is also useful to illustrate the remarks presented in Section II-C. To that end, a representative node, namely node 4 , is selected.

Remarks 1 and 3, which are respectively related to $N_{s}^{R P}$ and $D_{s}^{R P}$, rely on the knowledge of the branches supplying the demand at each node from the substation. As can be seen in Fig. 1, the demand at node 4 is supplied from the substation through branches 1-2 and 2-4. Thus, according to Remark 1, the expected rate of repair-and-switching interruptions affecting node $4, N_{4}^{R P}$, is equal to the sum of the failure rates of those branches, i.e., $0.5+0.1=0.6$ interruptions/year. Analogously, as per Remark 3, the expected duration of repair-and-switching interruptions affecting node $4, D_{4}^{R P}$, is equal to the sum over those branches of the products of the corresponding failure rate and repair-andswitching interruption duration, i.e., $0.5 \times 1+0.1 \times 4=0.9$ h/year.

Remark 2 deals with $N_{s}^{S W}$. As shown in Fig. 1, the only branch in feeder 1 that is not used to supply the demand at node 4 is branch 2-3. Therefore, based on Remark $2, N_{4}^{S W}$ is equal to $\lambda_{23}$, i.e., 0.2 interruptions/year. Moreover, since node 4 belongs to feeder 1 comprising branches 1-2, 2-3, and 2-4, the total expected rate of interruptions affecting node $4, N_{4}^{T}$, is equal to $\lambda_{12}+\lambda_{23}+\lambda_{24}$, i.e., $N_{4}^{T}=0.5+0.2+0.1=$ 0.8 interruptions/year. Thus, according to Remark $2, N_{4}^{S W}$ can also be computed as $N_{4}^{T}-N_{4}^{R P}=0.8-0.6=0.2$ interruptions/year.

Remark 4 is associated with $D_{s}^{S W}$. As above mentioned, branch 2-3 is the only branch in feeder 1 that is not used to supply the demand at node 4 . Therefore, based on Remark 4, $D_{4}^{S W}$ is equal to $\lambda_{23} \tau_{23}^{S W}$, i.e., $0.2 \times 0.5=0.1 \mathrm{~h}$ /year. Note that $D_{4}^{S W}$ is equal to the difference between $D_{4}^{T, S W}=$ $\lambda_{12} \tau_{12}^{S W}+\lambda_{23} \tau_{23}^{S W}+\lambda_{24} \tau_{24}^{S W}$ and $\bar{D}_{4}^{S W}=\lambda_{12} \tau_{12}^{S W}+$ $\lambda_{24} \tau_{24}^{S W}$. Numerically, $D_{4}^{T, S W}=0.5 \times 0.15+0.2 \times 0.50+0.1 \times$ $0.25=0.2 \mathrm{~h} /$ year and $\bar{D}_{4}^{S W}=0.5 \times 0.15+0.1 \times 0.25=0.1$ $\mathrm{h} /$ year, thereby giving rise to $D_{4}^{S W}=0.2-0.1=0.1 \mathrm{~h} /$ year.

The application of this process to the other load nodes yields the results listed in columns $8-11$ of Table I.

\section{Proposed Optimization-BAsed Approach}

This section presents a two-step approach that allows computing in an exact way $N_{s}^{R P}, N_{s}^{S W}, D_{s}^{R P}$, and $D_{s}^{S W}$ without using simulation. The first step involves the solution of a set of linear programs. As a result, the optimal values of a set of binary-valued continuous decision variables allow representing the relationship between nodal interruption rates, branch failure rates, and duration rates set forth in Remarks 14. The second step relies on equivalent algebraic expressions for $N_{s}^{R P}, N_{s}^{S W}, D_{s}^{R P}$, and $D_{s}^{S W}$ in terms of the optimal values of those decision variables. The proposed approach, albeit illustrated with the radial system of Fig. 1, is suitable not only for radial networks but also for practical meshed and radially-operated distribution networks, thereby paving the way for its incorporation in reliability-constrained operational and planning models. This section concludes with a discussion on the steps required for such potential applicability.

\section{A. Step 1: Linear Program for Topology Characterization}

The topological information required to compute $N_{s}^{R P}$, $N_{s}^{S W}, D_{s}^{R P}$, and $D_{s}^{S W}$ as per Remarks $1-4$ can be modeled by the outcomes of a set of optimal power flows, one per load node, for a fictitious system with the same radial topology of the original radially-operated network and with a constant power factor across the network. Each optimal power flow models the operation of the fictitious system under a loading condition characterized by one nodal demand equal to $1 \mathrm{pu}$ whereas the remaining nodal demands are set at 0 . For each load node $s \in \Psi^{L N}$, the proposed optimal power flow is formulated as follows:

$\underset{\tilde{f}_{i j s}, \tilde{g}_{i s}^{S S}}{\operatorname{Minimize}} \sum_{(i, j) \in \Upsilon}\left(\tilde{f}_{i j s}+\tilde{f}_{j i s}\right)$

subject to:

$$
\begin{aligned}
& \sum_{j \in \Psi_{i}}\left(\tilde{f}_{i j s}-\tilde{f}_{j i s}\right)=\tilde{g}_{i s}^{S S}-\tilde{L}_{i s} ; \forall i \in \Psi^{N} \\
& 0 \leq \tilde{f}_{i j s} \leq 1 ; \forall i \in \Psi_{j}, \forall j \in \Psi^{N} \\
& 0 \leq \tilde{g}_{i s}^{S S} \leq 1 ; \forall i \in \Psi^{S S}
\end{aligned}
$$


where $\tilde{L}_{i s}= \begin{cases}1 ; & \forall i \in \Psi^{L N} \mid i=s \\ 0 ; & \text { otherwise. }\end{cases}$

In (11)-(14), branch-related variables $\tilde{f}_{i j s}$ and substationrelated variables $\tilde{g}_{i s}^{S S}$ represent the fictitious system operation under the condition associated with load node $s$. The goal of the proposed model is the minimization of the sum of all branch-related variables $\tilde{f}_{i j s}$ (11) subject to nodal power balance equations (12), limits on branch-related variables (13), and limits on substation-related variables (14). Expressions (12) extend the distribution flow model presented in [22] for a radial network by considering two continuous variables per branch, $\tilde{f}_{i j s}$ and $\tilde{f}_{j i s}$, in order to model the direction of the fictitious power flow under each operating condition. Note that, for the condition associated with node $s, \tilde{f}_{i j s}$ is equal to $1 \mathrm{pu}$, i.e., the fictitious power flow through the branch connecting nodes $i$ and $j$ measured at node $i$, only when the fictitious power flows from $i$ to $j$, being 0 otherwise. The interested reader is referred to [21], [23], where this two-variable-based distribution load flow model was successfully applied within a different context.

The constraint set (12)-(14) features a unimodular matrix structure (see [24], proposition 3.2) and parameters $\tilde{L}_{i s}$ are binary. As a consequence, continuous variables $\tilde{f}_{i j s}$ and $\tilde{g}_{i s}^{S S}$ can only take two values, namely 0 and 1 . In addition, for each branch $i-j$ and load node $s$, the optimization goal (11) precludes $\tilde{f}_{i j s}$ and $\tilde{f}_{j i s}$ from being simultaneously equal to 1 . As an example, the application of (11)-(14) to the network depicted in Fig. 1 yields the results shown in Fig. 2, where variables $\tilde{f}_{i j s}$ with optimal values equal to 1 are represented only.

The solution to (11)-(14) is particularly useful for the purposes of reliability assessment of the original system. It is worth emphasizing that the optimal values for branch-related variables $\tilde{f}_{i j s}$ allow identifying the branches that are used to supply the nodal demands from the substation node as well as the direction of the power flows for the original system. Thus, if $\tilde{f}_{i j s}=1$ or $\tilde{f}_{j i s}=1$ the branch connecting nodes $i$ and $j$ is used in the original system to supply the demand at node $s$. On the other hand, if $\tilde{f}_{i j s}=\tilde{f}_{j i s}=0$ the branch connecting nodes $i$ and $j$ is not used to supply the demand at node $s$. Moreover, if $\tilde{f}_{i j s}=1$ the direction of the corresponding flow is from node $i$ to node $j$.

In other words, the network topology is explicitly characterized by the optimal values of $\tilde{f}_{i j s}$. Moreover, the node-branch relationship described in Remarks $1-4$ is readily provided as follows:

- If $\tilde{f}_{i j s}+\tilde{f}_{j i s}=1$, node $s$ is downstream of branch $i$ $j$ and would thus be affected by a repair-and-switching interruption should such a branch experience a fault. Thus, the branches $i-j$ used to supply the demand at node $s$ from the substation are those for which $\tilde{f}_{i j s}+\tilde{f}_{j i s}$ is equal to 1 . This result is useful to calculate $N_{s}^{R P}$ and $D_{s}^{R P}$ as per Remarks 1 and 3 .

- For a branch $i$ - $j$ with a breaker, $\tilde{f}_{i j s}+\tilde{f}_{j i s}=1$ implies that node $\underset{\sim}{s}$ belongs to the feeder protected by this breaker, whereas $\tilde{f}_{i j s}+\tilde{f}_{j i s}=0$ means that node $s$ belongs to a different feeder. Additionally, for a branch $r-s, \tilde{f}_{r s s}$
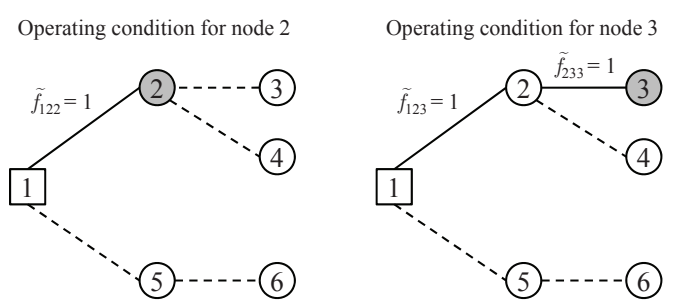

Operating condition for node 4

Operating condition for node 5
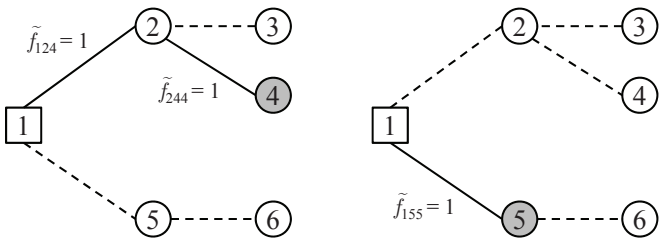

Operating condition for node 6
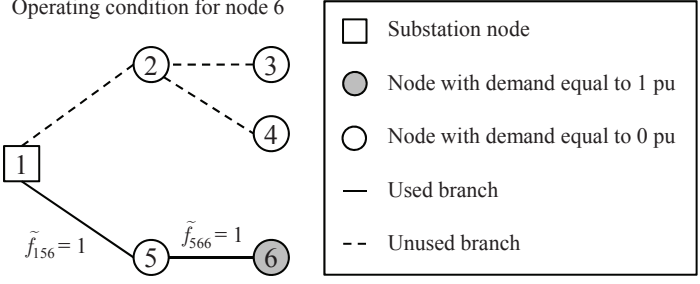

Fig. 2. Operation of the fictitious system for the illustrative example.

is equal to 1 if the demand at node $s$ is injected by this branch, being 0 otherwise. Both results are useful to calculate $N_{s}^{S W}$ and $D_{s}^{S W}$ as per Remarks 2 and 4.

We recognize that an equivalent model relying on a single variable per branch and without substation-related variables, $\tilde{g}_{i s}^{S S}$, can be derived to mathematically represent the topology of a radial network. However, such a simpler model would not be suitable for operational and planning models dealing with practical meshed and radially-operated networks supplied by more than one substation, for which the proposed tool is intended.

\section{B. Step 2: Calculation of Expected Nodal Interruption Rates and Durations}

As described next, and based on the above findings, the expressions for $N_{s}^{R P}, N_{s}^{S W}, D_{s}^{R P}$, and $D_{s}^{S W}$ are equivalently cast in terms of $\tilde{f}_{i j s}$. Thus, reliability indices can be computed without requiring a simulation-based algorithm, which constitutes the main contribution of this paper. For a comprehensive explanation, the derivation of the expressions for $N_{s}^{S W}$ and $D_{s}^{S W}$ relies on auxiliary expressions in terms of $N_{s}^{T}, N_{i j}^{C B}$, $D_{s}^{T, S W}, D_{i j}^{C B, S W}$, and $\bar{D}_{s}^{S W}$. As done for the conventional algorithm, the example analyzed in Section II-D is used to illustrate the proposed expressions for $N_{s}^{R P}, N_{s}^{S W}, D_{s}^{R P}$, and $D_{s}^{S W}$.

1) Calculation of $N_{s}^{R P}$ : As explained above, if $\tilde{f}_{i j s}=1$ or $\tilde{f}_{j i s}=1$ branch $i$ - $j$ is used to supply the demand at node $s$ from the substation node. Thus, as per Remark 1, branchrelated variables $\tilde{f}_{i j s}$ are useful to flag whether the outage of branch $i$ - $j$ yields a repair-and-switching interruption for the load at node $s$. Hence, the expected rate of nodal repair-andswitching interruptions can be formulated as: 
$N_{s}^{R P}=\sum_{(i, j) \in \Upsilon}\left(\tilde{f}_{i j s}+\tilde{f}_{j i s}\right) \lambda_{i j} ; \forall s \in \Psi^{L N}$.

For example, node 4 experiences repair-and-switching interruptions under the outages of branches 1-2 and 2-4 (Table I). As can be seen in Fig. 2, $\tilde{f}_{124}=1$ and $\tilde{f}_{244}=1$. According to (15) and the optimal solutions for $\tilde{f}_{i j s}$ shown in Fig. 2, the expected rate of repair-and-switching interruptions for load node 4 is calculated as follows:

$$
\begin{aligned}
N_{4}^{R P} & =\left(\tilde{f}_{124}+\tilde{f}_{214}\right) \lambda_{12}+\left(\tilde{f}_{234}+\tilde{f}_{324}\right) \lambda_{23} \\
& +\left(\tilde{f}_{244}+\tilde{f}_{424}\right) \lambda_{24}+\left(\tilde{f}_{154}+\tilde{f}_{514}\right) \lambda_{15} \\
& +\left(\tilde{f}_{564}+\tilde{f}_{654}\right) \lambda_{56}=(1+0) \times 0.5+(0+0) \times 0.2 \\
& +(1+0) \times 0.1+(0+0) \times 0.3+(0+0) \times 0.4 \\
& =0.6 \text { interruptions/year. }
\end{aligned}
$$

2) Calculation of $N_{s}^{S W}$ : According to Remark 2, for each load node, the difference between the total expected rate of interruptions and the expected rate of repair-and-switching interruptions yields the expected rate of switching-only interruptions:

$N_{s}^{S W}=N_{s}^{T}-N_{s}^{R P} ; \forall s \in \Psi^{L N}$.

From Remark 2, the total expected rate of interruptions affecting node $s, N_{s}^{T}$, is equal to the expected rate of interruptions affecting the circuit breaker in the corresponding feeder, $N_{i j}^{C B}$. Thus, the relationship between $N_{s}^{T}$ and $N_{i j}^{C B}$ can be formulated as follows:

$N_{s}^{T}=\sum_{i \in \Psi^{S S}} \sum_{j \in \Psi_{i}} N_{i j}^{C B}\left(\tilde{f}_{i j s}+\tilde{f}_{j i s}\right) ; \forall s \in \Psi^{L N}$.

Furthermore, based on Remark 2, $N_{i j}^{C B}$ is determined by the sum of the failure rates of the branches belonging to the feeder protected by the corresponding breaker. Thus, $N_{i j}^{C B}$ can be expressed as follows:

$N_{i j}^{C B}=\sum_{s \in \Psi^{L N}}\left[\left(\sum_{r \in \Psi_{s}} \tilde{f}_{r s s} \lambda_{r s}\right) \tilde{f}_{i j s}\right] ; \forall i \in \Psi^{S S}, \forall j \in \Psi_{i}$

where $\lambda_{r s}$ is equal to $\lambda_{s r}$.

In the outer summation of (19), the use of $\tilde{f}_{i j s}$ is intended to exclusively account for the nodes belonging to the feeder protected by the breaker under consideration. Analogously, in the inner summation, the correct branch is identified by $\tilde{f}_{r s s}$.

Using (15), (18), and (19) in (17) yields:

$$
\begin{aligned}
N_{s}^{S W} & =\sum_{i \in \Psi^{S S}} \sum_{j \in \Psi_{i}} \sum_{s^{\prime} \in \Psi^{L N}}\left[\left(\sum_{r \in \Psi_{s^{\prime}}} \tilde{f}_{r s^{\prime} s^{\prime}} \lambda_{r s^{\prime}}\right) \tilde{f}_{i j s^{\prime}}\right]\left(\tilde{f}_{i j s}+\tilde{f}_{j i s}\right) \\
& -\sum_{(i, j) \in \Upsilon}\left(\tilde{f}_{i j s}+\tilde{f}_{j i s}\right) \lambda_{i j} ; \forall s \in \Psi^{L N} .
\end{aligned}
$$

For illustration purposes, the above expressions for $N_{i j}^{C B}$, $N_{s}^{T}$, and $N_{s}^{S W}$ are applied to the six-node system depicted in Fig. 1. From Fig. 2, it can be observed that, under the conditions defined by nodes 2,3 , and 4 , which are in the same feeder as circuit breaker B1, the variables representing the fictitious flows through the branches injecting power at such nodes, i.e., $\tilde{f}_{122}, \tilde{f}_{233}$, and $\tilde{f}_{244}$, are all equal to 1 . Similarly, the conditions associated with nodes 5 and 6, under the protection of circuit breaker B2, yield $\tilde{f}_{155}=\tilde{f}_{566}=1$. The particularization of (19) for the circuit breakers located at branches 1-2 and 1-5 of the illustrative example gives rise to:

$$
\begin{aligned}
N_{12}^{C B} & =\left(\tilde{f}_{122} \lambda_{12}+\tilde{f}_{322} \lambda_{32}+\tilde{f}_{422} \lambda_{42}\right) \tilde{f}_{122} \\
& +\left(\tilde{f}_{233} \lambda_{23}\right) \tilde{f}_{123}+\left(\tilde{f}_{244} \lambda_{24}\right) \tilde{f}_{124} \\
& +\left(\tilde{f}_{155} \lambda_{15}+\tilde{f}_{655} \lambda_{65}\right) \tilde{f}_{125}+\left(\tilde{f}_{566} \lambda_{56}\right) \tilde{f}_{126} \\
& =(1 \times 0.5+0 \times 0.2+0 \times 0.1) \times 1 \\
& +(1 \times 0.2) \times 1+(1 \times 0.1) \times 1 \\
& +(1 \times 0.3+0 \times 0.4) \times 0+(1 \times 0.4) \times 0 \\
& =0.8 \text { interruptions/year } \\
N_{15}^{C B} & =\left(\tilde{f}_{122} \lambda_{12}+\tilde{f}_{322} \lambda_{32}+\tilde{f}_{422} \lambda_{42}\right) \tilde{f}_{152} \\
& +\left(\tilde{f}_{233} \lambda_{23}\right) \tilde{f}_{153}+\left(\tilde{f}_{244} \lambda_{24}\right) \tilde{f}_{154} \\
& +\left(\tilde{f}_{155} \lambda_{15}+\tilde{f}_{655} \lambda_{65}\right) \tilde{f}_{155}+\left(\tilde{f}_{566} \lambda_{56}\right) \tilde{f}_{156} \\
& =(1 \times 0.5+0 \times 0.2+0 \times 0.1) \times 0 \\
& +(1 \times 0.2) \times 0+(1 \times 0.1) \times 0 \\
& +(1 \times 0.3+0 \times 0.4) \times 1+(1 \times 0.4) \times 1 \\
& =0.7 \text { interruptions/year. }
\end{aligned}
$$

As an example, for load node 4, the total expected rate of interruptions is calculated using (18) as follows:

$$
\begin{aligned}
N_{4}^{T} & =N_{12}^{C B}\left(\tilde{f}_{124}+\tilde{f}_{214}\right)+N_{15}^{C B}\left(\tilde{f}_{154}+\tilde{f}_{514}\right) \\
& =0.8 \times(1+0)+0.7 \times(0+0) \\
& =0.8 \text { interruptions/year. }
\end{aligned}
$$

Hence, using the results of (16) and (23) in (17), the expected rate of switching-only interruptions for node 4 can be obtained as follows:

$$
\begin{aligned}
N_{4}^{S W} & =N_{4}^{T}-N_{4}^{R P}=0.8-0.6 \\
& =0.2 \text { interruptions/year. }
\end{aligned}
$$

3) Calculation of $D_{s}^{R P}$ : Based on Remark 3 and the relationship between branch outages and nodal repair-andswitching interruptions modeled by variables $\tilde{f}_{i j s}$, the expected duration of nodal repair-and-switching interruptions can be formulated as:

$D_{s}^{R P}=\sum_{(i, j) \in \Upsilon}\left(\tilde{f}_{i j s}+\tilde{f}_{j i s}\right) \lambda_{i j} \tau_{i j}^{R P} ; \forall s \in \Psi^{L N}$.

Using the optimal solutions for $\tilde{f}_{i j s}$ (Fig. 2) in (25), the expected duration of repair-and-switching interruptions for load node 4 is calculated as follows:

$$
\begin{aligned}
D_{4}^{R P} & =\left(\tilde{f}_{124}+\tilde{f}_{214}\right) \lambda_{12} \tau_{12}^{R P}+\left(\tilde{f}_{234}+\tilde{f}_{324}\right) \lambda_{23} \tau_{23}^{R P} \\
& +\left(\tilde{f}_{244}+\tilde{f}_{424}\right) \lambda_{24} \tau_{24}^{R P}+\left(\tilde{f}_{154}+\tilde{f}_{514}\right) \lambda_{15} \tau_{15}^{R P} \\
& +\left(\tilde{f}_{564}+\tilde{f}_{654}\right) \lambda_{56} \tau_{56}^{R P}=(1+0) \times 0.5 \times 1.0 \\
& +(0+0) \times 0.2 \times 2.0+(1+0) \times 0.1 \times 4.0 \\
& +(0+0) \times 0.3 \times 1.5+(0+0) \times 0.4 \times 3.0 \\
& =0.9 \mathrm{~h} \text { /year. }
\end{aligned}
$$


4) Calculation of $D_{s}^{S W}$ : According to Remark 4, the expected duration of nodal switching-only interruptions is given by:

$D_{s}^{S W}=D_{s}^{T, S W}-\bar{D}_{s}^{S W} ; \forall s \in \Psi^{L N}$.

Similar to $D_{s}^{R P}, \bar{D}_{s}^{S W}$ can be expressed in terms of $\tilde{f}_{i j s}$ as follows:

$\bar{D}_{s}^{S W}=\sum_{(i, j) \in \Upsilon}\left(\tilde{f}_{i j s}+\tilde{f}_{j i s}\right) \lambda_{i j} \tau_{i j}^{S W} ; \forall s \in \Psi^{L N}$.

In addition, $D_{s}^{T, S W}$ can be cast as:

$D_{s}^{T, S W}=\sum_{i \in \Psi_{S S}} \sum_{j \in \Psi_{i}} D_{i j}^{C B, S W}\left(\tilde{f}_{i j s}+\tilde{f}_{j i s}\right) ; \forall s \in \Psi^{L N}$

where the breaker-related duration, $D_{i j}^{C B, S W}$, is expressed as follows:

$D_{i j}^{C B, S W}=\sum_{s \in \Psi^{L N}}\left[\left(\sum_{r \in \Psi_{s}} \tilde{f}_{r s s} \lambda_{r s} \tau_{r s}^{S W}\right) \tilde{f}_{i j s}\right]$;

$\forall i \in \Psi^{S S}, \forall j \in \Psi_{i}$

where $\tau_{r s}^{S W}$ is equal to $\tau_{s r}^{S W}$.

Similar to (19), in (30), the nodes belonging to the feeder protected by the circuit breaker under consideration are identified by $\tilde{f}_{i j s}$ whereas the use of $\tilde{f}_{r s s}$ accounts for the correct branch.

Hence, the desired expression of $D_{s}^{S W}$ in terms of $\tilde{f}_{i j s}$ is given by the use of (28)-(30) in (27):

$$
\begin{aligned}
D_{s}^{S W} & =\sum_{i \in \Psi^{S S}} \sum_{j \in \Psi_{i}} \sum_{s^{\prime} \in \Psi^{L N}}\left[\left(\sum_{r \in \Psi_{s^{\prime}}} \tilde{f}_{r s^{\prime} s^{\prime}} \lambda_{r s^{\prime}} \tau_{r s^{\prime}}^{S W}\right) \tilde{f}_{i j s^{\prime}}\right]\left(\tilde{f}_{i j s}+\tilde{f}_{j i s}\right) \\
& -\sum_{(i, j) \in \Upsilon}\left(\tilde{f}_{i j s}+\tilde{f}_{j i s}\right) \lambda_{i j} \tau_{i j}^{S W} ; \forall s \in \Psi^{L N} .
\end{aligned}
$$

As an example, using (28) for load node 4 yields:

$$
\begin{aligned}
\bar{D}_{4}^{S W} & =\left(\tilde{f}_{124}+\tilde{f}_{214}\right) \lambda_{12} \tau_{12}^{S W}+\left(\tilde{f}_{234}+\tilde{f}_{324}\right) \lambda_{23} \tau_{23}^{S W} \\
& +\left(\tilde{f}_{244}+\tilde{f}_{424}\right) \lambda_{24} \tau_{24}^{S W}+\left(\tilde{f}_{154}+\tilde{f}_{514}\right) \lambda_{15} \tau_{15}^{S W} \\
& +\left(\tilde{f}_{564}+\tilde{f}_{654}\right) \lambda_{56} \tau_{56}^{S W}=(1+0) \times 0.5 \times 0.15 \\
& +(0+0) \times 0.2 \times 0.5+(1+0) \times 0.1 \times 0.25 \\
& +(0+0) \times 0.3 \times 0.2+(0+0) \times 0.4 \times 0.6 \\
& =0.1 \mathrm{~h} \text { /year. }
\end{aligned}
$$

Moreover, the particularization of (30) for the circuit breakers located at branches 1-2 and 1-5 of the illustrative example gives rise to:

$$
\begin{aligned}
D_{12}^{C B, S W} & =\left(\tilde{f}_{122} \lambda_{12} \tau_{12}^{S W}+\tilde{f}_{322} \lambda_{32} \tau_{32}^{S W}+\tilde{f}_{422} \lambda_{42} \tau_{42}^{S W}\right) \tilde{f}_{122} \\
& +\left(\tilde{f}_{233} \lambda_{23} \tau_{23}^{S W}\right) \tilde{f}_{123}+\left(\tilde{f}_{244} \lambda_{24} \tau_{24}^{S W}\right) \tilde{f}_{124} \\
& +\left(\tilde{f}_{155} \lambda_{15} \tau_{15}^{S W}+\tilde{f}_{655} \lambda_{65} \tau_{65}^{S W}\right) \tilde{f}_{125} \\
& +\left(\tilde{f}_{566} \lambda_{56} \tau_{56}^{S W}\right) \tilde{f}_{126}=(1 \times 0.5 \times 0.15 \\
& +0 \times 0.2 \times 0.5+0 \times 0.1 \times 0.25) \times 1 \\
& +(1 \times 0.2 \times 0.5) \times 1+(1 \times 0.1 \times 0.25) \times 1 \\
& +(1 \times 0.3 \times 0.2+0 \times 0.4 \times 0.6) \times 0 \\
& +(1 \times 0.4 \times 0.6) \times 0=0.2 \mathrm{~h} / \text { year }
\end{aligned}
$$

$$
\begin{aligned}
D_{15}^{C B, S W} & =\left(\tilde{f}_{122} \lambda_{12} \tau_{12}^{S W}+\tilde{f}_{322} \lambda_{32} \tau_{32}^{S W}+\tilde{f}_{422} \lambda_{42} \tau_{42}^{S W}\right) \tilde{f}_{152} \\
& +\left(\tilde{f}_{233} \lambda_{23} \tau_{23}^{S W}\right) \tilde{f}_{153}+\left(\tilde{f}_{244} \lambda_{24} \tau_{24}^{S W}\right) \tilde{f}_{154} \\
& +\left(\tilde{f}_{155} \lambda_{15} \tau_{15}^{S W}+\tilde{f}_{655} \lambda_{65} \tau_{65}^{S W}\right) \tilde{f}_{155} \\
& +\left(\tilde{f}_{566} \lambda_{56} \tau_{56}^{S W}\right) \tilde{f}_{156}=(1 \times 0.5 \times 0.15 \\
& +0 \times 0.2 \times 0.5+0 \times 0.1 \times 0.25) \times 0 \\
& +(1 \times 0.2 \times 0.5) \times 0+(1 \times 0.1 \times 0.25) \times 0 \\
& +(1 \times 0.3 \times 0.2+0 \times 0.4 \times 0.6) \times 1 \\
& +(1 \times 0.4 \times 0.6) \times 1=0.3 \mathrm{~h} / \text { year. }
\end{aligned}
$$

Both results (33), (34) allow computing $D_{s}^{T, S W}$ for all load nodes. For the representative node 4, expression (29) gives rise to:

$$
\begin{aligned}
D_{4}^{T, S W} & =D_{12}^{C B, S W}\left(\tilde{f}_{124}+\tilde{f}_{214}\right)+D_{15}^{C B, S W}\left(\tilde{f}_{154}+\tilde{f}_{514}\right) \\
& =0.2 \times(1+0)+0.3 \times(0+0)=0.2 \mathrm{~h} / \text { year. }
\end{aligned}
$$

Finally, from (27), the expected duration of switching-only interruptions for node 4 can be obtained as follows:

$D_{4}^{S W}=D_{4}^{T, S W}-\bar{D}_{4}^{S W}=0.2-0.1=0.1 \mathrm{~h} /$ year.

\section{Potential Applicability}

The ultimate goal of the proposed approach is to allow the incorporation of analytical expressions for reliability assessment in the formulation of distribution system operational and planning models, thereby enabling the application of solution methodologies different from presently used heuristics and metaheuristics. Such incorporation would require the following modifications in current optimization models: 1) extending the set of decision variables to include $D_{s}^{R P}, D_{s}^{S W}, \tilde{f}_{i j s}$, $\tilde{g}_{i s}^{S S}, N_{s}^{R P}$, and $\left.N_{s}^{S W} ; 2\right)$ extending the constraint set to include new expressions built on (12)-(15), (20), (25), and (31); and 3) expressing the reliability cost in terms of the newly added decision variables. Note that the minimization of the reliability cost in the resulting reliability-constrained models would prevent $\tilde{f}_{i j s}$ and $\tilde{f}_{j i s}$ from being simultaneously equal to 1 for each branch $i-j$ and load node $s$, thereby having the same effect as (11).

The above extension of the problem formulation would give rise to nonlinearities due to the cross products of newly added variables $\tilde{f}_{i j s}$ in (20) and (31). Note, however, that, given the fact that variables $\tilde{f}_{i j s}$ are binary valued, such bilinear terms can be converted into equivalent linear expressions using well-known integer algebra results without needing additional binary variables [25]. Hence, although the incorporation of reliability requires a larger number of variables and constraints, the computational complexity would not be drastically increased. The interested reader is referred to the Appendix for a general formulation of the resulting optimization problems.

\section{NUMERICAL RESULTS}

Results from several case studies are presented in this section. The proposed approach has been first applied to a 37-node test system. Additionally, in order to assess the scalability of the proposed methodology, four larger case 


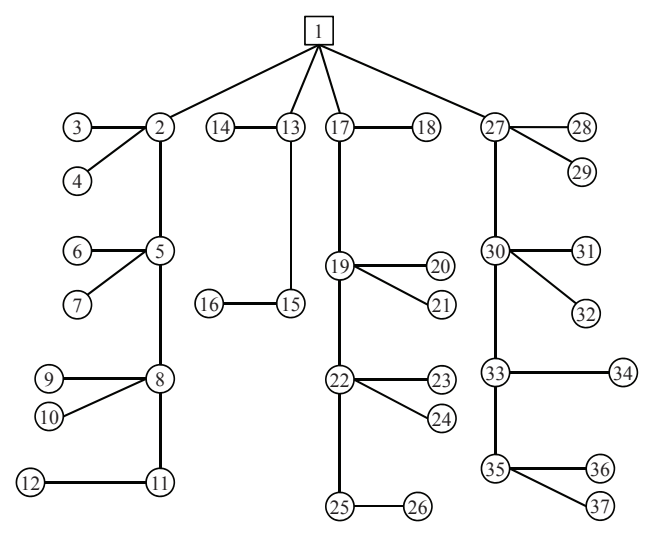

Fig. 3. One-line diagram of the 37-node system.

TABLE II

37-Node System - Nodal Peak Demands (MW)

\begin{tabular}{cccccccc}
\hline $\begin{array}{c}\text { Node } \\
s\end{array}$ & $L_{s}$ & Node & \multirow{2}{*}{$L_{s}$} & Node & \multirow{2}{*}{ Node } & \multirow{2}{*}{$L_{s}$} \\
\hline 2 & 0.99 & 11 & 2.43 & 20 & 1.60 & 29 & 0.18 \\
3 & 2.01 & 12 & 1.18 & 21 & 0.20 & 30 & 1.55 \\
4 & 0.41 & 13 & 1.46 & 22 & 2.02 & 31 & 2.06 \\
5 & 0.36 & 14 & 1.71 & 23 & 0.41 & 32 & 0.26 \\
6 & 1.49 & 15 & 2.06 & 24 & 3.04 & 33 & 1.65 \\
7 & 1.57 & 16 & 2.74 & 25 & 2.08 & 34 & 1.87 \\
8 & 0.91 & 17 & 1.78 & 26 & 1.69 & 35 & 2.32 \\
9 & 1.45 & 18 & 2.67 & 27 & 1.92 & 36 & 1.38 \\
10 & 1.98 & 19 & 2.30 & 28 & 1.44 & 37 & 0.89 \\
\hline
\end{tabular}

studies including a real-life distribution system with 1080 nodes have been solved.

Simulations have been implemented on a Dell Precision M4800 laptop with an Intel ${ }^{\circledR}$ Core $^{\mathrm{TM}}$ i7-4910MQ processor at $2.9 \mathrm{GHz}$ and $16 \mathrm{~GB}$ of RAM using CPLEX 12.6 [26] and GAMS 24.2 [27]. All case studies were solved to optimality by setting the optimality tolerance of CPLEX equal to 0 .

\section{A. 37-Node Case Study}

The first benchmark is a $13.2-\mathrm{kV}$ test system based on that described in [20]. As shown in Fig. 3, the system comprises 36 load nodes, represented by circles, and 1 substation node, depicted as a square. Base power and base voltage of the system are 1 MVA and $13.2 \mathrm{kV}$, respectively. Nodal peak demands and the number of customers per node are listed in Tables II and III, respectively. The loading condition is modeled by three load levels with loading factors equal to $70 \%, 83 \%$, and $100 \%$ of the corresponding peak demand, and durations equal to $2000 \mathrm{~h} /$ year, $5760 \mathrm{~h} /$ year, and $1000 \mathrm{~h} /$ year, respectively. Branch data are provided in Table IV. Based on [8], all branches are characterized by 0.1 failures per year and $\mathrm{km}$. Therefore, branch failure rates are set so that $\lambda_{i j}=0.1 \ell_{i j}$.

The proposed optimization-based approach required 4.15 s to achieve the values for $N_{s}^{R P}, N_{s}^{S W}, D_{s}^{R P}$, and $D_{s}^{S W}$ reported in Table $\mathrm{V}$, which are identical to those provided by the conventional simulation-based algorithm [7] outlined in Section II-B. As can be seen in Table V, the expected rate and duration of nodal repair-and-switching interruptions generally grow as the distance to the substation increases, whereas the expected rate and duration of nodal switchingonly interruptions experience, in general, the opposite result.
TABLE III

37-Node System - Number of Customers Per Node

\begin{tabular}{|c|c|c|c|c|c|c|c|}
\hline $\begin{array}{c}\text { Node } \\
s\end{array}$ & $N C_{s}$ & $\begin{array}{c}\text { Node } \\
s\end{array}$ & $N C_{s}$ & $\begin{array}{c}\text { Node } \\
s\end{array}$ & $N C_{s}$ & $\begin{array}{c}\text { Node } \\
s\end{array}$ & $N C_{s}$ \\
\hline 2 & 177 & 11 & 208 & 20 & 235 & 29 & 198 \\
\hline 3 & 214 & 12 & 260 & 21 & 140 & 30 & 222 \\
\hline 4 & 222 & 13 & 305 & 22 & 222 & 31 & 168 \\
\hline 5 & 166 & 14 & 303 & 23 & 220 & 32 & 268 \\
\hline 6 & 214 & 15 & 140 & 24 & 217 & 33 & 245 \\
\hline 7 & 294 & 16 & 153 & 25 & 231 & 34 & 200 \\
\hline 8 & 302 & 17 & 286 & 26 & 196 & 35 & 270 \\
\hline 9 & 171 & 18 & 251 & 27 & 193 & 36 & 258 \\
\hline 10 & 279 & 19 & 144 & 28 & 271 & 37 & 227 \\
\hline
\end{tabular}

TABLE IV

37-NODE SYSTEM - BRANCH DATA

\begin{tabular}{|c|c|c|c|c|c|c|c|}
\hline $\begin{array}{c}\text { Branch } \\
i-j\end{array}$ & $\begin{array}{c}\ell_{i j} \\
(\mathrm{~km})\end{array}$ & $\begin{array}{c}\tau_{i j}^{R P} \\
(\mathrm{~h})\end{array}$ & $\begin{array}{c}\tau_{i j}^{S W} \\
(\mathrm{~h})\end{array}$ & $\begin{array}{c}\text { Branch } \\
i-j\end{array}$ & $\begin{array}{c}\ell_{i j} \\
(\mathrm{~km})\end{array}$ & $\begin{array}{c}\tau_{i j}^{R P} \\
\text { (h) }\end{array}$ & $\begin{array}{c}\tau_{i j}^{S W} \\
\text { (h) }\end{array}$ \\
\hline $1-2$ & 3.52 & 1.90 & 0.25 & $17-19$ & 2.35 & 2.07 & 0.24 \\
\hline $1-13$ & 1.57 & 2.02 & 0.28 & $19-20$ & 1.54 & 2.13 & 0.29 \\
\hline $1-17$ & 1.52 & 1.70 & 0.28 & $19-21$ & 1.78 & 1.64 & 0.25 \\
\hline $1-27$ & 3.85 & 1.54 & 0.27 & $19-22$ & 1.82 & 2.07 & 0.29 \\
\hline $2-3$ & 1.16 & 2.38 & 0.26 & $22-23$ & 1.57 & 2.41 & 0.29 \\
\hline $2-4$ & 1.56 & 2.17 & 0.27 & $22-24$ & 1.65 & 2.49 & 0.21 \\
\hline $2-5$ & 2.03 & 2.27 & 0.22 & $22-25$ & 2.10 & 1.65 & 0.22 \\
\hline $5-6$ & 1.16 & 2.06 & 0.24 & $25-26$ & 1.06 & 2.10 & 0.21 \\
\hline $5-7$ & 1.56 & 2.20 & 0.28 & $27-28$ & 1.25 & 1.83 & 0.27 \\
\hline $5-8$ & 2.12 & 1.93 & 0.25 & $27-29$ & 1.54 & 1.97 & 0.26 \\
\hline $8-9$ & 1.76 & 1.99 & 0.30 & $27-30$ & 2.03 & 2.25 & 0.24 \\
\hline $8-10$ & 1.93 & 1.69 & 0.28 & $30-31$ & 1.33 & 1.96 & 0.25 \\
\hline $8-11$ & 1.60 & 1.52 & 0.23 & $30-32$ & 1.80 & 2.09 & 0.22 \\
\hline $11-12$ & 2.16 & 2.43 & 0.23 & $30-33$ & 1.99 & 1.51 & 0.24 \\
\hline $13-14$ & 0.90 & 2.49 & 0.23 & $33-34$ & 2.12 & 2.16 & 0.22 \\
\hline $13-15$ & 3.15 & 2.43 & 0.23 & $33-35$ & 1.44 & 1.76 & 0.20 \\
\hline $15-16$ & 1.14 & 1.69 & 0.27 & $35-36$ & 1.63 & 1.76 & 0.23 \\
\hline $17-18$ & 1.24 & 1.97 & 0.26 & $35-37$ & 1.82 & 2.10 & 0.26 \\
\hline
\end{tabular}

The results provided in Table $\mathrm{V}$ allow computing the standard reliability indices. Nodal reliability indices CIF and CID are listed in Table VI. From this table, two results are remarkable: 1) load nodes belonging to longer feeders feature larger customer interruption frequencies, and 2) the customer interruption duration increases with the distance to the substation. In addition, system reliability indices SAIFI and SAIDI are respectively equal to 1.81 interruptions/year and $1.53 \mathrm{~h} /$ year, whereas ASAI is equal to $99.98 \%$ and EENS is $69.51 \mathrm{MWh} /$ year.

\section{B. Real-Sized Case Studies}

The scalability of the proposed approach has been validated with four case studies comprising 85, 137, 417, and 1080 nodes, which are based on those described in [28]-[31], respectively. For reproducibility purposes, data for the four case studies can be downloaded from [32]. Table VII summarizes the results achieved by the simulation-based algorithm and the proposed equivalent approach. As can be seen, using an optimization-based framework requires longer computing times than the simulation-based algorithm, ranging between $8.36 \mathrm{~s}$ for the 85 -node system and $365.95 \mathrm{~s}$ for the 1080node system. Notwithstanding, it is worth emphasizing that the computational effort associated with the proposed approach is moderate bearing in mind that the four test systems are real-sized and that simulations have been implemented on a modest laptop. Therefore, this is a promising result for 
TABLE V

37-NODE SYSTEM - RESULTS FOR $N_{s}^{R P}$ AND $N_{s}^{S W}$ (interruptions/year), and $D_{s}^{R P}$ and $D_{s}^{S W}$ (h/year)

\begin{tabular}{|c|c|c|c|c|c|c|c|c|c|c|c|c|c|c|c|c|c|c|c|}
\hline Node & $N_{s}^{R P}$ & $N_{s}^{S W}$ & $D_{s}^{R P}$ & $D_{s}^{S W}$ & Node & $N_{s}^{R P}$ & $N_{s}^{S W}$ & $D_{s}^{R P}$ & $D_{s}^{S W}$ & Node & $N_{s}^{R P}$ & $N_{s}^{S W}$ & $D_{s}^{R P}$ & $D_{s}^{S W}$ & Node & $N_{s}^{R P}$ & $N_{s}^{S W}$ & $D_{s}^{R P}$ & $D_{s}^{S W}$ \\
\hline 2 & 0.35 & 1.70 & 0.67 & 0.43 & 11 & 0.93 & 1.13 & 1.78 & 0.30 & 20 & 0.54 & 1.12 & 1.07 & 0.28 & 29 & 0.54 & 1.54 & 0.90 & 0.36 \\
\hline 3 & 0.47 & & 0.94 & & & & & & & & & & 1. & & & & & & 0.35 \\
\hline 4 & 0.51 & 1.55 & 1.01 & 0.3 & & 0.16 & 0.52 & 0.32 & 0.12 & 2 & 0.57 & 1.09 & 1.12 & 0.27 & 31 & 0.72 & 1.36 & 31 & 0.32 \\
\hline 5 & 0.56 & & 1.13 & & 1 & 0.2 & 0.4 & 0.5 & 0.1 & 2 & 0.7 & 0. & 1. & 0.2 & 3 & 0. & & 43 & 0.32 \\
\hline 6 & 0.67 & 1.39 & 1.37 & 0.36 & 15 & 0.47 & 0.20 & 1.08 & 0.05 & 24 & 0.73 & 0.93 & 1.53 & 0.2 & 33 & 0.79 & 1.29 & 1.35 & 0.31 \\
\hline 7 & 0.71 & & 1.47 & & 16 & 0.59 & & & & 25 & & & & & & 1.00 & & 1.81 & 0.26 \\
\hline 8 & & & & & & & & 0.2 & & 2 & & & & & & 93 & & & 0.28 \\
\hline 9 & 0.94 & 1.11 & 1.89 & 0.28 & 18 & 0.28 & 1.3 & 0.50 & 0.3 & 27 & 0.3 & 1.7 & 0.5 & 0.4 & 36 & 1.09 & 0.99 & 1.89 & 0.24 \\
\hline 10 & 0.96 & 1.10 & 1.86 & 0.28 & 19 & 0.39 & 1.28 & 0.74 & 0.32 & 28 & 0.51 & 1.57 & 0.82 & 0.37 & 37 & 1.11 & 0.97 & 1.99 & 0.23 \\
\hline
\end{tabular}

TABLE VI

37-NODE SYSTEM - RESULTS FOR $C I F_{s}$ (interruptions/year) and $C I D_{s}$ (h/year)

\begin{tabular}{cccccccccccc}
\hline Node & $C I F_{s}$ & $C I D_{s}$ & Node & $C I F_{s}$ & $C I D_{s}$ & Node & $C I F_{s}$ & $C I D_{s}$ & Node & $C I F_{s}$ & $C I D_{s}$ \\
\hline 2 & 2.06 & 1.10 & 11 & 2.06 & 2.08 & 20 & 1.66 & 1.35 & 29 & 2.08 & 1.26 \\
3 & 2.06 & 1.35 & 12 & 2.06 & 2.56 & 21 & 1.66 & 1.32 & 30 & 2.08 & 1.40 \\
4 & 2.06 & 1.40 & 13 & 0.68 & 0.44 & 22 & 1.66 & 1.39 & 31 & 2.08 & 1.63 \\
5 & 2.06 & 1.52 & 14 & 0.68 & 0.64 & 23 & 1.66 & 1.72 & 32 & 2.08 & 1.74 \\
6 & 2.06 & 1.73 & 15 & 0.68 & 1.13 & 24 & 1.66 & 1.77 & 33 & 2.08 & 1.66 \\
7 & 2.06 & 1.82 & 16 & 0.68 & 1.30 & 25 & 1.66 & 1.69 & 34 & 2.08 & 2.07 \\
8 & 2.06 & 1.88 & 17 & 1.66 & 0.64 & 26 & 1.66 & 1.89 & 35 & 2.08 & 1.88 \\
9 & 2.06 & 2.17 & 18 & 1.66 & 0.85 & 27 & 2.08 & 1.00 & 36 & 2.08 & 2.13 \\
10 & 2.06 & 2.15 & 19 & 1.66 & 1.07 & 28 & 2.08 & 1.19 & 37 & 2.08 & 2.22 \\
\hline
\end{tabular}

TABLE VII

RESULTS FOR LARGE-SCALE SYSTEMS

\begin{tabular}{lrrrr}
\cline { 2 - 5 } & \multicolumn{4}{c}{ Number of nodes } \\
\cline { 2 - 5 } & \multicolumn{1}{c}{85} & \multicolumn{1}{c}{137} & \multicolumn{1}{c}{417} & \multicolumn{1}{c}{1080} \\
\hline Time for the simulation-based algorithm (s) & 0.03 & 0.08 & 0.64 & 14.07 \\
Time for the proposed approach (s) & 8.36 & 16.27 & 59.80 & 365.95 \\
SAIFI (interruptions/year) & 1.97 & 1.79 & 1.67 & 1.99 \\
SAIDI (h/year) & 2.41 & 1.65 & 0.99 & 1.21 \\
ASAI (\%) & 99.97 & 99.98 & 99.99 & 99.99 \\
EENS (MWh/year) & 48.90 & 48.13 & 91.16 & 106.53 \\
\hline
\end{tabular}

the subsequent integration of the linear-programming-based formulation in reliability-constrained optimization models for distribution systems. Table VII also reports the results for several relevant system reliability indices, which, as expected, are identical for both methodologies.

\section{Conclusions}

This paper has pushed forward the notion that simulationbased reliability assessment can be equivalently performed by an optimization-based approach relying on the solution of a set of linear programs. Numerical results show the equivalence between the proposed approach and the conventional simulationbased algorithm. Moreover, the moderate computational effort required for large-scale test systems comprising up to 1080 nodes reveals the computational effectiveness of the proposed approach.

It is worth emphasizing that the main contribution of this paper, namely the development of algebraic expressions for reliability assessment where the topology is represented through decision variables of an optimization process, will pave the way for the use of non-heuristic solution methodologies to effectively address the integration of reliability assessment in the optimization models used for the operation and planning of distribution systems. Such integration constitutes our ongoing research.
Another interesting avenue of research is the extension of the approach to consider practical modeling aspects of utmost importance within the context of active distribution networks such as distributed generation and post-fault network reconfiguration to restore the service for load nodes downstream of the fault. Based on our recent work [21], we are currently investigating the inclusion of generation at the distribution level via modeling distributed generators as negative loads. However, we recognize that extending the model to characterize the above issues needs further research effort and numerical studies. Research will also be conducted to explicitly model the effect of temporary faults, protection failures, transient disturbances, and line overloading.

\section{APPENDIX}

The incorporation of the proposed analytical expressions for reliability assessment in the formulation of distribution optimization models can be cast in a compact way as follows:

$\underset{\mathbf{x}, \mathbf{y}, \mathbf{z}}{\operatorname{Minimize}} c^{I-O}(\mathbf{x}, \mathbf{y})+c^{R}(\mathbf{z})$

subject to:

$\mathbf{x} \in\{0,1\}^{n}$

$f(\mathbf{x}, \mathbf{y}) \leq 0$

$g(\mathbf{x}, \mathbf{z}) \leq 0$

where $\mathbf{x}$ is the vector of binary variables modeling investment and operating decisions; $\mathbf{y}$ is the vector of continuous variables also related to the operation and planning of distribution systems; $\mathbf{z}$ is the vector of the newly added variables relating topology decisions and reliability, i.e., $D_{s}^{R P}, D_{s}^{S W}, \tilde{f}_{i j s}, \tilde{g}_{i s}^{S S}$, $N_{s}^{R P}$, and $N_{s}^{S W} ; c^{I-O}(\mathbf{x}, \mathbf{y})$ is the investment and operating $\operatorname{cost} ; c^{R}(\mathbf{z})$ is the reliability cost; $n$ is the dimension of vector $\mathbf{x} ; f(\mathbf{x}, \mathbf{y})$ represents the set of constrained functions typically used to model the operation and planning of distribution systems; and $g(\mathbf{x}, \mathbf{z})$ is a new set of constrained functions. 
The objective function to be minimized (37) consists of two terms respectively related to the investment and operating cost and the reliability cost. The binary nature of $\mathbf{x}$ is imposed in (38). Conventional investment and operational constraints are modeled in (39). Finally, the relationship between $\mathbf{x}$ and $\mathbf{z}$ is formulated in (40). Note that such a relationship is built upon the newly developed expressions (12)-(15), (20), (25), and (31). Thus, expressions (37) and (40) constitute the main differences between the resulting problems and current reliability-constrained distribution optimization models. As a major salient aspect, the reliability cost is explicitly expressed in terms of the newly added decision variables included in $\mathbf{z}$, which, in turn, allow representing the implicit dependence of the reliability cost on the network topology.

\section{REFERENCES}

[1] H. L. Willis, Power Distribution Planning Reference Book, 2nd ed. New York, NY, USA: Marcel Dekker, Inc., 2004.

[2] A. A. Chowdhury and D. O. Koval, Power Distribution System Reliability. Practical Methods and Applications. Hoboken, NJ, USA: John Wiley \& Sons, Inc., 2009.

[3] R. Billinton and J. E. Billinton, "Distribution system reliability indices," IEEE Trans. Power Deliv., vol. 4, no. 1, pp. 561-568, Jan. 1989.

[4] R. Billinton and R. N. Allan, Reliability Evaluation of Power Systems. New York, NY, USA: Plenum Press, 1996.

[5] IEEE Guide for Electric Power Distribution Reliability Indices, IEEE Standard 1366-2003, May 2004.

[6] A. A. Chowdhury and D. O. Koval, "Current practices and customer value-based distribution system reliability planning," IEEE Trans. Ind. Appl., vol. 40, no. 5, pp. 1174-1182, Sep./Oct. 2004.

[7] R. E. Brown, Electric Power Distribution Reliability, 2nd ed. Boca Raton, FL, USA: CRC Press, 2008.

[8] R. C. Lotero and J. Contreras, "Distribution system planning with reliability," IEEE Trans. Power Deliv., vol. 26, no. 4, pp. 2552-2562, Oct. 2011.

[9] R. E. Brown and A. P. Hanson, "Impact of two-stage service restoration on distribution reliability," IEEE Trans. Power Syst., vol. 16, no. 4, pp. 624-629, Nov. 2001.

[10] S. Kalambe and G. Agnihotri, "Loss minimization techniques used in distribution network: Bibliographical survey," Renew. Sust. Energ. Rev. vol. 29, pp. 184-200, Jan. 2014.

[11] P. S. Georgilakis and N. D. Hatziargyriou, "A review of power distribution planning in the modern power systems era: Models, methods and future research," Electr. Power Syst. Res., vol. 121, pp. 89-100, Apr. 2015.

[12] Y. Tang, "Power distribution system planning with reliability modeling and optimization," IEEE Trans. Power Syst., vol. 11, no. 1, pp. 181-189, Feb. 1996.

[13] A. A. Chowdhury and D. O. Koval, "Value-based distribution system reliability planning," IEEE Trans. Ind. Appl., vol. 34, no. 1, pp. 23-29, Jan./Feb. 1998.

[14] G. Celli, E. Ghiani, S. Mocci, and F. Pilo, "A multiobjective evolutionary algorithm for the sizing and siting of distributed generation," IEEE Trans. Power Syst., vol. 20, no. 2, pp. 750-757, May 2005.

[15] J.-H. Teng, Y.-H. Liu, C.-Y. Chen, and C.-F. Chen, "Value-based distributed generator placements for service quality improvements," Int. J. Electr. Power Energy Syst., vol. 29, no. 3, pp. 268-274, Mar. 2007.

[16] E. G. Carrano, F. G. Guimarães, R. H. C. Takahashi, O. M. Neto, and F. Campelo, "Electric distribution network expansion under loadevolution uncertainty using an immune system inspired algorithm," IEEE Trans. Power Syst., vol. 22, no. 2, pp. 851-861, May 2007.

[17] V. F. Martins and C. L. T. Borges, "Active distribution network integrated planning incorporating distributed generation and load response uncertainties," IEEE Trans. Power Syst., vol. 26, no. 4, pp. 2164-2172, Nov. 2011.

[18] I. Ziari, G. Ledwich, A. Ghosh, and G. Platt, "Optimal distribution network reinforcement considering load growth, line loss, and reliability," IEEE Trans. Power Syst., vol. 28, no. 2, pp. 587-597, May 2013.

[19] M. E. Samper and A. Vargas, "Investment decisions in distribution networks under uncertainty with distributed generation-Part I: Model formulation," IEEE Trans. Power Syst., vol. 28, no. 3, pp. 2331-2340, Aug. 2013.
[20] - "Investment decisions in distribution networks under uncertainty with distributed generation-Part II: Implementation and results," IEEE Trans. Power Syst., vol. 28, no. 3, pp. 2341-2351, Aug. 2013.

[21] G. Muñoz-Delgado, J. Contreras, and J. M. Arroyo, "Multistage generation and network expansion planning in distribution systems considering uncertainty and reliability," IEEE Trans. Power Syst., vol. 31, no. 5, pp. 3715-3728, Sep. 2016

[22] S. Haffner, L. F. A. Pereira, L. A. Pereira, and L. S. Barreto, "Multistage model for distribution expansion planning with distributed generationPart I: Problem formulation," IEEE Trans. Power Deliv., vol. 23, no. 2, pp. 915-923, Apr. 2008.

[23] G. Muñoz-Delgado, J. Contreras, and J. M. Arroyo, "Joint expansion planning of distributed generation and distribution networks," IEEE Trans. Power Syst., vol. 30, no. 5, pp. 2579-2590, Sep. 2015.

[24] L. Wolsey, Integer Programming. New York, NY, USA: Wiley InterScience, 1998

[25] C. A. Floudas, Nonlinear and Mixed-Integer Optimization: Fundamentals and Applications. New York, NY, USA: Oxford University Press, 1995.

[26] IBM ILOG CPLEX, 2016. [Online]. Available: http://www-01.ibm. $\mathrm{com} /$ software/commerce/optimization/cplex-optimizer

[27] GAMS Development Corporation, 2016. [Online]. Available: http:// www.gams.com

[28] C.-T. Su and C.-S. Lee, "Network reconfiguration of distribution systems using improved mixed-integer hybrid differential evolution," IEEE Trans. Power Deliv., vol. 18, no. 3, pp. 1022-1027, Jul. 2003.

[29] M. Lavorato, M. J. Rider, A. V. Garcia, and R. Romero, "A constructive heuristic algorithm for distribution system planning," IEEE Trans. Power Syst., vol. 25, no. 3, pp. 1734-1742, Aug. 2010.

[30] M. Lavorato, J. F. Franco, M. J. Rider, and R. Romero, "Imposing radiality constraints in distribution system optimization problems," IEEE Trans. Power Syst., vol. 27, no. 1, pp. 172-180, Feb. 2012.

[31] Laboratório de Planejamento de Sistemas de Energia Elétrica, "1080 buses test system." [Online]. Available: http://www.feis.unesp.br/\#!/departamentos/engenharia-eletrica/ pesquisas-e-projetos/lapsee/downloads/materiais-de-cursos1193/

[32] G. Muñoz-Delgado, J. Contreras, and J. M. Arroyo, "Reliability assessment for distribution optimization models: A non-simulationbased linear programming approach-Data for test systems." [Online]. Available: https://drive.google.com/file/d/0B5Wja2Owv_ j4cFdLUWxSTVU0Ylk/view?usp=sharing

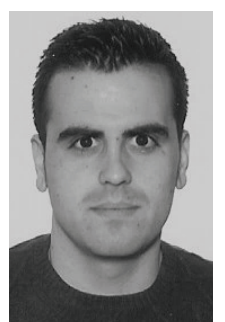

Gregorio Muñoz-Delgado (S'14) received the Ingeniero Industrial degree and the M.Sc. degree from the Universidad de Castilla-La Mancha, Ciudad Real, Spain, in 2012 and 2013, respectively. He is currently pursuing the Ph.D. degree at the Universidad de Castilla-La Mancha.

His research interests are in the fields of power systems planning, operations, and economics.

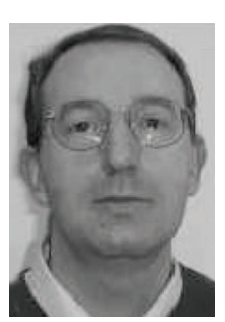

Javier Contreras (SM'05-F'15) received the B.S degree in electrical engineering from the University of Zaragoza, Zaragoza, Spain, in 1989, the M.Sc. degree from the University of Southern California, Los Angeles, CA, USA, in 1992, and the Ph.D. degree from the University of California, Berkeley, CA, USA, in 1997.

$\mathrm{He}$ is a Professor at the Universidad de Castilla-La Mancha, Ciudad Real, Spain. His research interests include power systems planning, operations, and economics, as well as electricity markets.

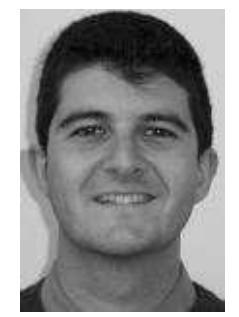

José M. Arroyo (S'96-M'01-SM'06) received the Ingeniero Industrial degree from the Universidad de Málaga, Málaga, Spain, in 1995, and the Ph.D. degree in power systems operations planning from the Universidad de Castilla-La Mancha, Ciudad Real, Spain, in 2000.

From June 2003 through July 2004, he held a Richard H. Tomlinson Postdoctoral Fellowship at the Department of Electrical and Computer Engineering of McGill University, Montreal, QC, Canada. He is currently a Full Professor of electrical engineering at the Universidad de Castilla-La Mancha. His research interests include operations, planning, and economics of power systems, as well as optimization. 